\title{
Molecular characterization and functional analysis of barley semi-dwarf mutant Riso no. 9265
}

Qiaojun Jia ${ }^{1,2}$, Chengdao Li $i^{3}$ Yi Shang ${ }^{2}$, Jinghuan Zhu², Wei Hua ${ }^{2}$ Junmei Wang ${ }^{2}$, Jianming Yang ${ }^{2 *}$ and Guoping Zhang ${ }^{1 *}$

\begin{abstract}
Background: $s d w 1 / d e n s o$ is one of the most important and useful semi-dwarf genes in barley breeding. At least four sdw1/denso alleles have been reported and $H_{v G A 20 o x_{2}}$ is considered as the candidate gene. Up to date, results of studies have not univocally proven the genetic relationship between sdw1/denso and HvGA200x . $_{2}$

Results: In the present study, a complete deletion of Morex_contig_40861 including both HVGA20ox 2 and Mloc_56463 genes was identified at the sdw 1 locus from a semi-dwarf mutant Riso no. 9265. Expression of the genes encoding gibberellin biosynthesis ( $H_{v G A 2 O O x_{1}}$ and $H_{v G A 3 O x_{2}}$ ) were increased in the mutant compared to the wild type Bomi, while the expression of GA catabolic gene HvGA2Ox 3 was decreased. Over-expression of $\mathrm{HVGA2OOX}_{2}$ could rescue the semi-dwarf phenotype and increase GAs concentration.

Conclusions: We confirmed that a GA biosynthetic enzyme HvGA200x 2 , acted as GA 20-oxidase, is the functional gene for the $s d w 1 / d e n s o$ semi-dwarfism. Lose of HVGA20ox 2 is partially compensated by HVGA20ox 1 and further feedback is regulated by gibberellin. We also deduced that the $s d w 1 / d e n s o$ allele itself affects later heading owing to its reduced endogenous GAs concentration.
\end{abstract}

Keywords: Barley, Deletion, Gene compensation, Gibberellin, Semi-dwarf

\section{Background}

Plant height is one of the most important agronomic traits in cereal crops that not only determines plant architecture, but also is closely associated with grain yield. A reduction in plant height usually leads to strong straw and high resistance to lodging, as happened in semi-dwarf rice and wheat cultivars. However, reduced plant height means lowering of canopy, which favors the epidemic spread of fungal diseases, resulting in an undesired increase of fungicide use, and a reduction of yield potential mainly due to smaller grain weight [1]. For optimizing the balance of plant height and yield, breeders have successfully utilized the semi-dwarf genes in cereal crop breeding since 1960s. The development and wide cultivation of semi-dwarf wheat and rice

\footnotetext{
* Correspondence: jmyang@163.com; zhanggp@zju.edu.cn

${ }^{2}$ Institute of Crop and Nuclear Technology Utilization, Zhejiang Academy of Agricultural Sciences, Hangzhou 310021, China

'Institute of Crop Science, Zhejiang University, Hangzhou 310058, China Full list of author information is available at the end of the article
}

varieties led to a dramatic increase of cereal production worldwide [2], which is labeled as a 'green revolution'.

The Green Revolution genes have been identified and isolated in the semi-dwarf varieties by reversed genetics. Among them, $s d 1$ in rice is one of the most famous genes and has been widely used to produce semi-dwarf phenotype in both japonica and indica rice. It was reported that $s d 1$ encoded one of the GA 20-oxidases and was involved in the last steps of gibberellin biosynthesis [3-6]. Semi-dwarf genes have also been extensively explored in barley breeding programs and more than 30 types of them have been described [7]. One of the most successfully used semi-dwarf genes in modern barley breeding is $s d w 1 / d e n s o$, which was postulated as homologous to ga5 in Arabidopsis and sd1 in rice [8, 9]. The main phenotypic effect of the $s d w 1 /$ denso gene is a $10-20 \mathrm{~cm}$ reduction of plant height, depending on environmental conditions [10]. Besides, there are some deleterious effects associated with $s d w 1 / d e n s o$ gene, such as late heading and 
maturity, decreased thousand-grain weight and high screening [10-15]. As for the relationship between grain yield and $s d w 1 /$ denso, both positive and negative were reported depending on different genetic backgrounds $[10,11,15]$. However, with its suitable semi-dwarf phenotype and potentially increased yield, $s d w 1 /$ denso has been introduced to numerous cultivars. For example, newly released more than 150 European cultivars carried the denso allele [16].

There are at least four independent alleles based on the allelism test done up to date [12, 17]. One spontaneous mutant was derived from a Danish variety Abed denso in 1946, as named accordingly [17]. Two alleles induced by X-ray with different parents were named as denso and $s d w 1$, respectively. Interestingly, the denso mutant has been used to develop malting barley, while the $s d w 1$ has been limited to feed barley $[10,12]$. The fourth $s d w 1$ allele was found in a $\mathrm{M}_{2}$ generation from the variety Bomi, treated by neutrons in the Stockholm reactor and named as Riso no. 9265. Although these mutants are characterized by semi-dwarf phenotype, all of them are sensitive to $\mathrm{GA}_{3}$ [18].

In 1993, $s d w 1 /$ denso was first mapped on the $3 \mathrm{HL}$ as a phenotypic trait [19]. In our previous study, GA20ox 2 homolog was identified using PCR method with the primers designed from the conserved domain of rice $s d 1$ and considered as a candidate gene of $s d w 1 /$ denso [8]. Meanwhile, it was found that the expression of HvGA20ox $x_{2}$ in denso mutant was reduced by 4 -fold, but almost 60 -fold in the $s d w 1$ mutant, compared to the control [15]. However, there was no direct evidence to prove the $H v G A 20 o x_{2}$ as the functional gene. In addition, the function of $H v G A 20 o x_{2}$ was predicted based on its functional domain, being highly similar with those of $s d 1$ $\left(\mathrm{OsGA20ox}_{2}\right)$ and ga5 (AtGA20ox $)_{1}$. No evidence showed its function in vitro or in vivo. In the present work we investigated the $s d w 1$ allele of Riso no. 9265 and identified a complete deletion contig, which includes two genes Mloc_56462 (HvGA20ox $)_{2}$ and Mloc_56463 (a putative methyltransferase PMT26). The function of the HvGA2Oox 2 gene was further analyzed using genetically-transformed Arabidopsis. Furthermore, realtime PCR assays revealed that transcript level of GA synthesis-related genes were significantly different between Bomi and Riso no. 9265. The current results showed that the semi-dwarf phenotype of Riso no. 9265 is attributed to the deletion of $H v G A 2 O O x_{2}$.

\section{Results}

Plant height, internode length and heading date of Bomi and Riso no. 9265

At maturity, plant height and length of spike, culm and internodes of Bomi and its semi-dwarf mutant Riso no. 9265 were measured (Table 1). Plant height of Bomi was
Table 1 Internode length and plant height of Bomi and Riso no. 9265

\begin{tabular}{lcccc}
\hline Traits & $\begin{array}{l}\text { Bomi } \\
\text { Length }(\mathrm{cm})\end{array}$ & $\%$ & $\begin{array}{l}\text { Riso no.9265 } \\
\text { Length }(\mathrm{cm})\end{array}$ & $\%$ \\
\hline Plant height & $83.2 \pm 1.1$ & & $63.5 \pm 2.4^{* *}$ & \\
Spike length & $10.1 \pm 0.4$ & & $10.0 \pm 0.5$ & \\
Culm height & $73.1 \pm 0.9$ & 100 & $53.5 \pm 2.1^{* *}$ & 100 \\
First-internode length & $25.2 \pm 1.0$ & 34.5 & $20.1 \pm 0.7^{*}$ & 37.6 \\
Second-internode length & $14.9 \pm 0.3$ & 20.4 & $13.1 \pm 0.7^{*}$ & 24.5 \\
Third-internode length & $10.5 \pm 0.4$ & 14.4 & $7.6 \pm 0.6^{* *}$ & 14.2 \\
Fourth-internode length & $10.8 \pm 0.4$ & 14.8 & $6.7 \pm 0.4^{* *}$ & 12.5 \\
Fifth-internode length & $8.2 \pm 0.6$ & 11.2 & $4.2 \pm 0.3^{* *}$ & 7.9 \\
Sixth-internode length & $3.5 \pm 0.6$ & 4.8 & $1.8 \pm 0.2^{* *}$ & 3.4 \\
\hline
\end{tabular}

Values are means $\pm \mathrm{SE}, \mathrm{N}=20$. ${ }^{*}$ significant difference at 0.005 level; ${ }^{*}$ significant difference at 0.001 level

$83.1 \mathrm{~cm}$, and Riso no. 9265 was $63.3 \mathrm{~cm}$, being only $76.2 \%$ of its wild type parent, as reported previously [17]. No obvious difference was found in spike length between the two genotypes. Obviously, the reduction of plant height for the mutant is completely attributed to the shorter culm length. Culm length of Riso no. 9265 was $53.5 \mathrm{~cm}$, being $19.6 \mathrm{~cm}$ shorter than that of Bomi. In fact, all internodes of the mutant were shorter than those of the wild type (Table 1). Moreover, the difference between the two genotypes was smaller for the length of the top two internodes and larger for the basal four internodes. Therefore, it may be assumed that the effect of the semi-dwarf gene in Riso no. 9265 is mainly on the basal internodes. In addition, Riso no. 9265 headed three days later than Bomi.

\section{HvGA20ox 2 was deleted in Riso no. 9265}

In this study, the complete genome sequence of HvGA20ox 2 (Mloc_56462) was identified after blast against Gramene (http://www.gramene.org/) using the sequences of $H \nu G A 20 o x_{2}$ from our previous study [8]. Two pairs of primers of $H \nu G A 20 o x_{2}$ were designed to amplify its genomic sequence. In Bomi, both primer pairs produced single band with different sizes. One is $2974 \mathrm{bp}$ and the other is $3165 \mathrm{bp}$. However, no PCR product was obtained in Riso no. 9265 (Fig. 1), indicating that $H v G A 20 o x_{2}$ is lost in the mutant. Thus, the sequences of $H v G A 20 o x_{2}$ were used to identify Contigs after blast against International Barley Genome Sequencing Consortium (http://webblast.ipk-gatersleben.de/bar ley/viroblast.php). One Morex_contig_40861, covering $21596 \mathrm{bp}$ was identified, and it contains $H v G A 20 o x_{2}$ and Mloc_56463 genes. Several primers covering Morex_ contig_40861 were designed (Additional file 1: Table S1) and PCR amplification succeeded in Bomi, but failed in the mutant Riso no. 9265. Furthermore, we were able to amplify flanking genes (Mloc_3247, Mloc_45089, 


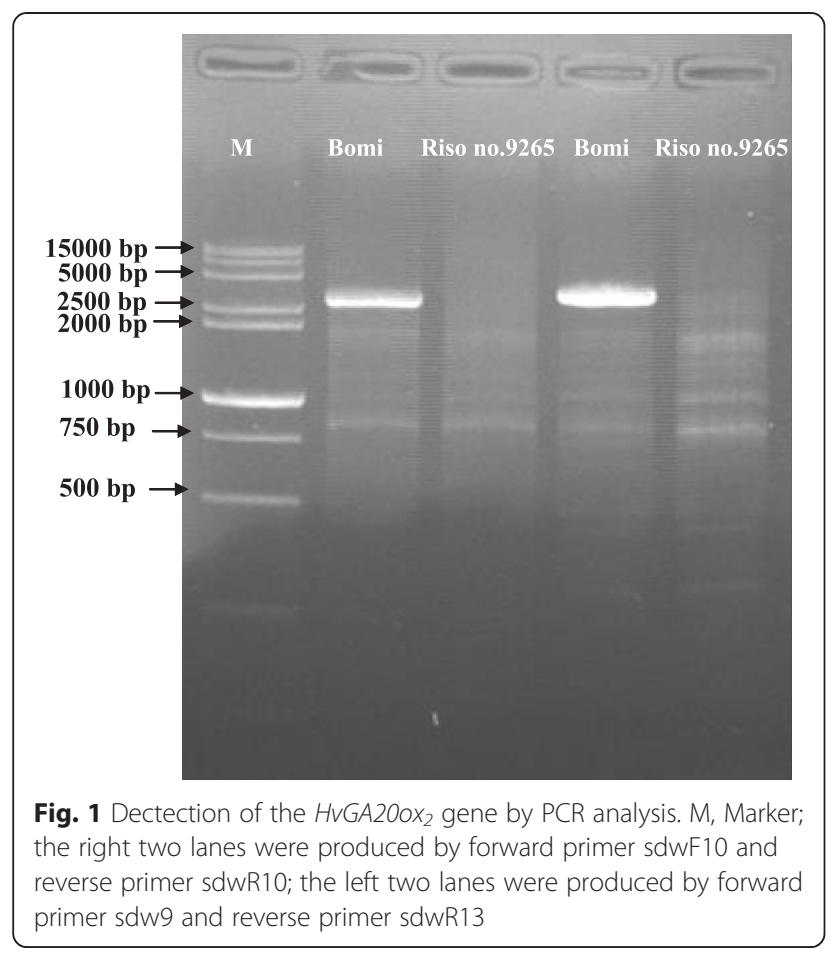

Mloc_51746 and Mloc_7311) of Morex_contig_40861, obtained using the latest barley assembly from Gramene and barley reference genome information from Leibniz Institute of Plant Genetics and Crop Plant Research (IPK) (primers are listed in Additional file 1: Table S1). As a result, it was found that there is a large deletion spanned at least two genes $H \nu G A 20 o x_{2}$ and Mloc_56463 in Riso no. 9265. A blast search for the Mloc_56463 protein sequence against NCBI identified a possible methyltransferase PMT26-like gene.

\section{Overexpression of $\mathrm{HvGA2Oox}_{2}$ caused more gibberellin production in Arabidopsis}

To evaluate if $H v G A 20 o x_{2}$ is responsible for the semidwarf phenotype, we examined transgenic Arabidopsis plants that over-express $H v G A 20 o x_{2}$ in wild type (Col-gl1) and a semi-dwarf mutant ga5-3. T1 transgenic plants were selected by BASTA analysis and PCR method. We obtained eleven and six transgenic lines in Col-gl1 and ga5-3 background, respectively. All of them displayed a higher growth rate. Homozygous T3 plants of transgenic Colgl1 lines (OE-1 and OE-2) and transgenic ga5-3 lines (OE-3 and OE-4) were taken randomly for further analysis. The four transgenic plants differed greatly in the expression levels of $H \nu G A 20 o x_{2}$. However, transcripts of barley GA oxidase-encoding gene were not found in both wild type and semi-dwarf mutant ga5-3 (Fig. 2).

The two independent transgenic lines $(\mathrm{OE}-1$ and $\mathrm{OE}-$ 2) showed GA-overdose phenotype although they differed in the expression level of $H v G A 20 o x_{2}$. There was no obvious difference in root length of 7-day-old seedlings between wild type and $H v G A 20 o x_{2}$ over-expression plants, but the latter had $50 \%$ longer hypocotyl than the former (Table 2 and Fig. 3). In addition, the transgenic plants flowered relatively early.

To determine whether the over-expression constructs would affect the phonotype of ga5-3, we characterized the GA-affecting traits in ga5-3 transgenic lines (OE-3 and OE-4), including hypocotyl length, internodes length and number, flowering time, and final plant height. The results showed that ga5-3 transgenic lines (OE-3 and OE-4) also had the GA-overdose phenotype, with longer hypocotyls and internodes, more internode number, flowering earlier in comparison with those of wild type

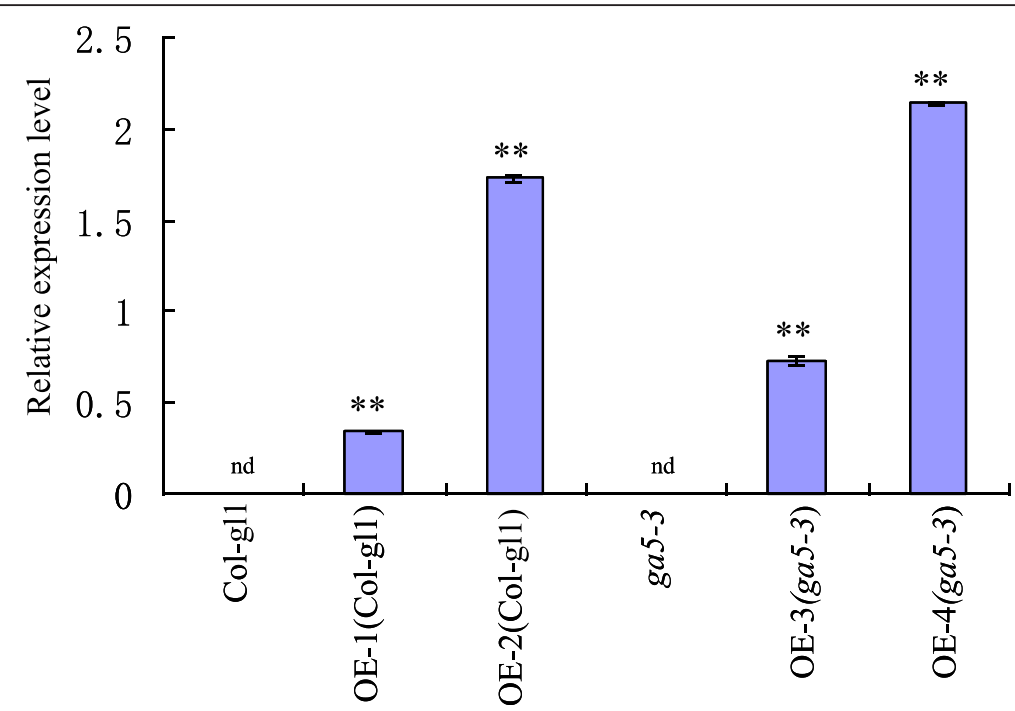

Fig. 2 Relative expression level of HvGA20ox 2 in transgenic Arabidopsis. nd, not detected. ${ }^{* *}$ means significant difference at 0.01 level. nd means not detected 
Table 2 Phenotypic parameters for wild type, ga5-3 and HvGA20ox 2 over-expressing transgenic Arabidopsis

\begin{tabular}{llccccc}
\hline Traits & Col-gl1 & OE-1(Col-gl1) & OE-2(Col-gl1) & ga5-3 & OE-3(ga5-3) & OE-4(ga5-3) \\
\hline Hypocotyl length $(\mathrm{mm})$ & $2.05 \pm 0.04$ & $3.64 \pm 0.10 \mathrm{~A}$ & $4.04 \pm 0.01 \mathrm{~A}$ & $2.07 \pm 0.04 \mathrm{~A}$ & $3.64 \pm 0.10 \mathrm{AB}$ & $3.75 \pm 0.12 \mathrm{AB}$ \\
Root length $(\mathrm{mm})$ & $36.1 \pm 0.7$ & $38.9 \pm 1.8$ & $40.1 \pm 1.2$ & $37.5 \pm 0.67$ & $40.3 \pm 1.4$ & $36.8 \pm 1.7$ \\
Flowering time $(\mathrm{d})$ & $26.2 \pm 0.35$ & $24.6 \pm 0.33 \mathrm{~A}$ & $23.0 \pm 0.25 \mathrm{~A}$ & $26.7 \pm 0.28$ & $22.5 \pm 0.23 \mathrm{AB}$ & $23.7 \pm 0.24 \mathrm{AB}$ \\
Vegetative internode length $(\mathrm{cm})$ & $1.9 \pm 0.1$ & $2.7 \pm 0.1 \mathrm{~A}$ & $2.9 \pm 0.1 \mathrm{~A}$ & $0.6 \pm 0.1 \mathrm{~A}$ & $3.0 \pm 0.2 \mathrm{AB}$ & $2.7 \pm 0.1 \mathrm{AB}$ \\
No.vegetative internodes & $3.4 \pm 0.1$ & $5.2 \pm 0.2 \mathrm{~A}$ & $5.0 \pm 0.2 \mathrm{~A}$ & $3.1 \pm 0.1 \mathrm{~A}$ & $4.8 \pm 0.4 \mathrm{AB}$ & $4.9 \pm 0.1 \mathrm{AB}$ \\
inflorescence internode length $(\mathrm{cm})$ & $0.61 \pm 0.01$ & $0.68 \pm 0.01 \mathrm{~A}$ & $0.71 \pm 0.02 \mathrm{~A}$ & $0.38 \pm 0.01 \mathrm{~A}$ & $0.67 \pm 0.02 \mathrm{AB}$ & $0.64 \pm 0.02 \mathrm{AB}$ \\
No.inflorescence internodes & $34.4 \pm 1.1$ & $47.3 \pm 1.2 \mathrm{~A}$ & $43.9 \pm 1.4 \mathrm{~A}$ & $31.5 \pm 1.1 \mathrm{~A}$ & $43.7 \pm 1.6 \mathrm{AB}$ & $47.3 \pm 1.9 \mathrm{AB}$ \\
Final plant $(\mathrm{cm})$ & $28.8 \pm 0.5$ & $46.8 \pm 0.7 \mathrm{~A}$ & $44.8 \pm 1.0 \mathrm{~A}$ & $14.6 \pm 0.4 \mathrm{~A}$ & $42.0 \pm 1.7 \mathrm{AB}$ & $43.8 \pm 1.1 \mathrm{AB}$ \\
\hline
\end{tabular}

The values are the means \pm SE

Capital letter A means significantly different from the wild type (Col-gl1); Capital letter B means significantly different from ga5-3 mutant
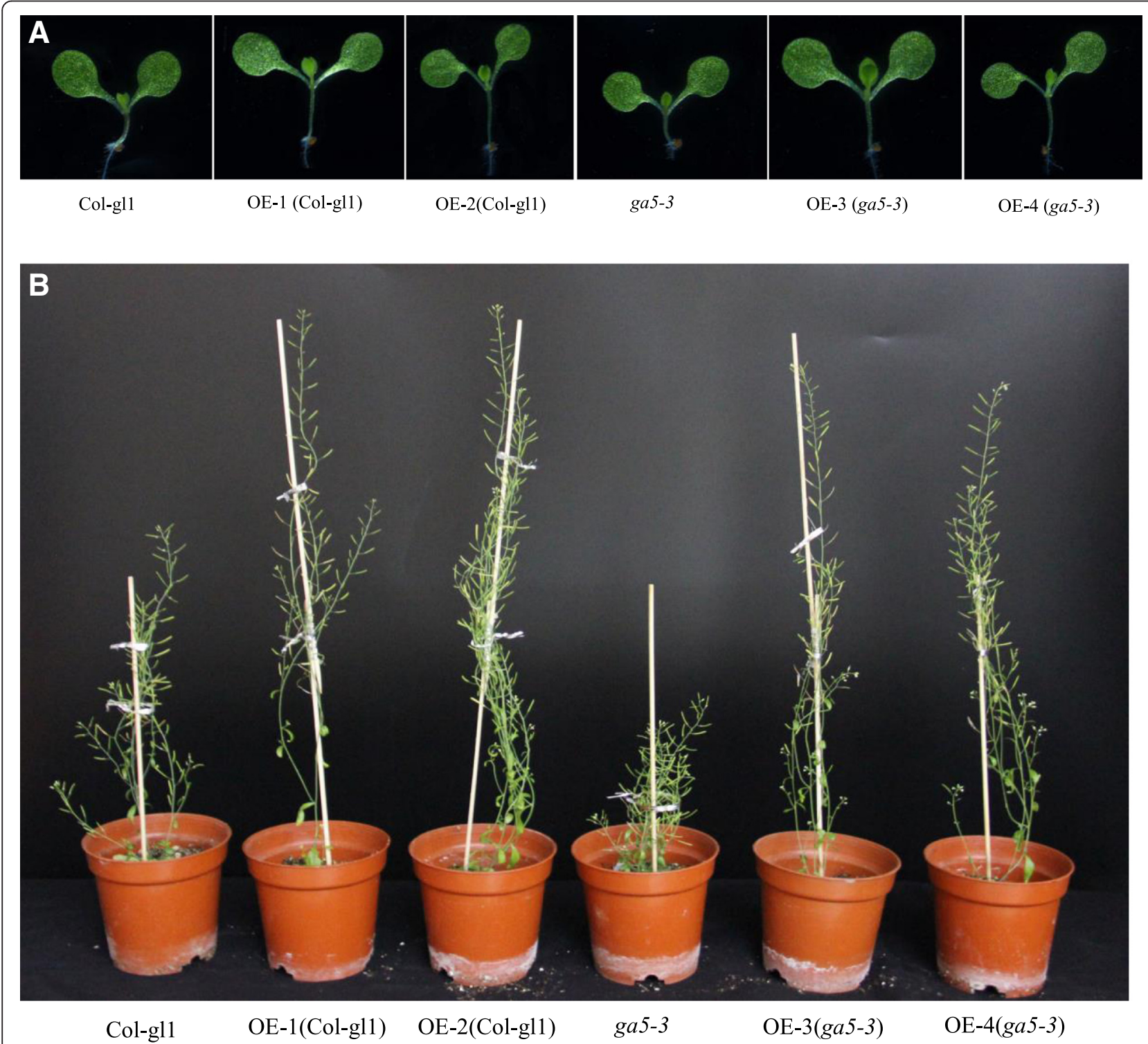

Fig. 3 Overexpression of HVGA200x in Arabidopsis affects plants development. The pictures compared the growth of transgenic and untransgenic at 1-week (a) and 6-week (b) 
or ga5-3 mutant (Table 2 and Fig. 3). It can be seen from Fig. 4 that there was the dramatic difference in plant height among the four transgenic lines, wild type and ga5-3 throughout the whole growth stage.

$\mathrm{GA}_{4+7}$ content were much higher than $\mathrm{GA}_{1+3}$ content in wild type. The transgenic plants had significantly higher $\mathrm{GA}_{1+3}$ content than both wild-type and ga5-3 plants, but the difference in $\mathrm{GA}_{4+7}$ content among them was much smaller. Moreover, $\mathrm{GA}_{1+3}$ content was lower in ga5-3 mutants than in wild-type plants, whereas both genotypes had the similar $\mathrm{GA}_{4+7}$ content (Fig. 5).

In order to determine whether the changes of phenotype and GA content observed in the transgenic lines are accompanied by the alteration in expression of GA biosynthesis and catabolism, three GA 20-oxidases, one GA 3-oxidase and one GA 2-oxidase, highly expressed in 7-day-old seedlings, were selected for further analysis. As shown in Fig. 6, the expression of $A t G A 20 o x_{2}$ and $A t G A 3 o x_{1}$ was greatly up-regulated in the $A t G A 20 o x_{1}$ deficient mutant ga5-3, while the expression of $A t G A 2 o x_{2}$ was significantly decreased in comparison with the wild type. No significant difference was found in the expression of $A t G A 20 o x_{3}$ between $g a 5-3$ and wild type. In the transgenic Arabidopsis with $H \nu G A 20 o x_{2}$ over-expression, the expression of $A t G A 20 o x_{1}, A t G A 20 o x_{2}$ and $A t G A 3 o x_{1}$ was distinctly decreased, however, $A t G A 2 o x_{2}$ was dramatically increased relative to wild type and ga5-3 mutants.

\section{The expression of GA biosynthesis and catabolism genes were changed in Riso no. 9265 mutant}

We determined the expression level of GA dioxygenase genes in stems of Bomi and Riso no. 9265 at the initial jointing stage. $H v G A 20 o x_{1}, H v G A 3 o x_{2}$ and $H v G A 2 o x_{3}$ showed high transcript level. The relative mRNA expression of $H \nu G A 200 x_{1}$ and $H \nu G A 3 o x_{2}$ were dramatically increased in the mutant Riso no. 9265 compared with those of Bomi. On the other hand, the mRNA expression of $H \nu G A 2 o x_{3}$ was decreased in the Riso no. 9265 (Fig. 7).

\section{Discussion}

Previously, we reported that barley $H v G A 20 o x_{2}$ (Mloc_56462) was a candidate gene of $s d w 1 /$ denso and different expression level of $H v G A 20 o x_{2}$ was observed between denso and $s d w 1$ (Jotun) alleles [8, 15]. Here, we reported that around $21 \mathrm{~kb}$ DNA fragment, including both $H v G A 20 o x_{2}$ and Mloc_56463 genes was completely deleted in the mutant Riso no. 9265. Because of the incomplete barley reference genomes and repetitive nature of the barley genome [20], the boundary of deleted segments was not determined precisely in Riso no. 9265. Gene annotation showed that $H \nu G A 20 o x_{2}$ has gibberellin 20-oxidase activity and may be involved in GA biosynthetic pathway. Moreover, the $s d w 1 /$ denso mutant is GA sensitive and exogenous application of $10 \mathrm{ppm} \mathrm{GA}_{3}$ restored its plant height [18], which indicates that $s d w 1 /$ denso mutants are GA deficiency. On the contrary, Mloc_56463 is a putative methyltransferase and has not been reported or predicted as a GA-related gene. Thus, $H v G A 20 o x_{2}$ is considered as the only candidate responsible for the semi-dwarfism in Riso no. 9265.

The GA biosynthetic pathway has been extensively investigated and the enzymes involved have been well characterized in plants [21]. The early GA-biosynthetic steps are encoded by single-copy genes, while the final steps catalyzed by GA 20-oxidase, GA 3-oxidase and GA 2-oxidase, are encoded by multigene families. GA20ox activity removes carbon-20 in the formation of C19-GA skeleton [22, 23]. In Arabidopsis, GA20-oxidase genes are differentially expressed and involved in different developmental processes controlled by GA, but the plants that constitutively express each of three GA20oxs (AtGA20ox $1, A t G A 20 o x_{2}$ and AtGA20ox $x_{3}$ ) behave as the control treated with GA [22, 23]. The GA-over-

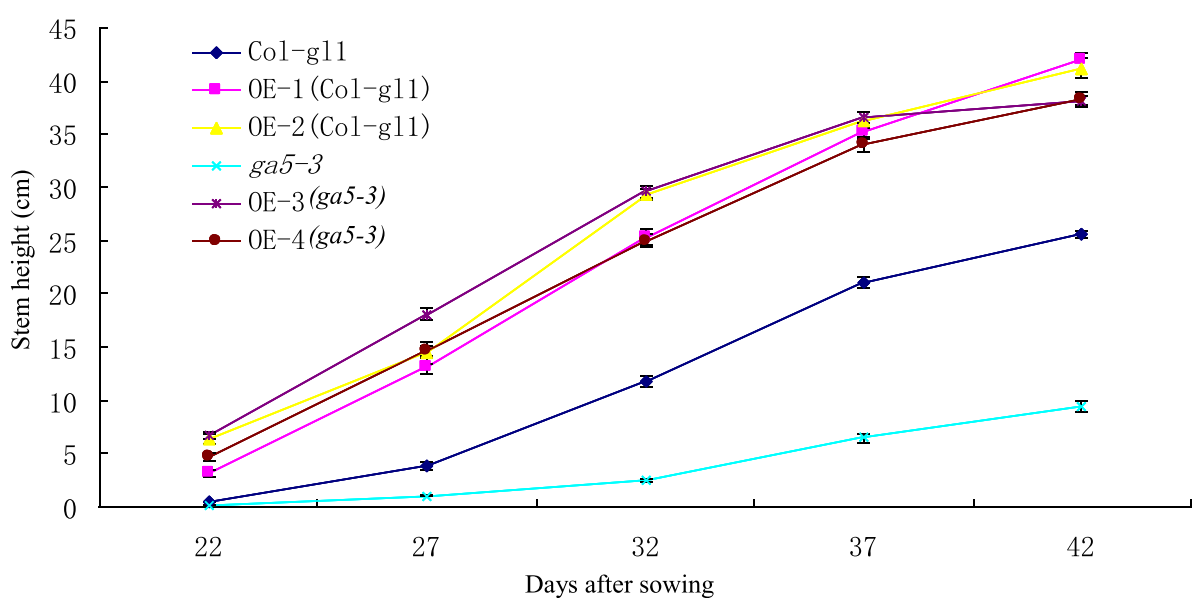

Fig. 4 Stem heights of Arabidopsis over expressing HvGA20ox 2 gene 


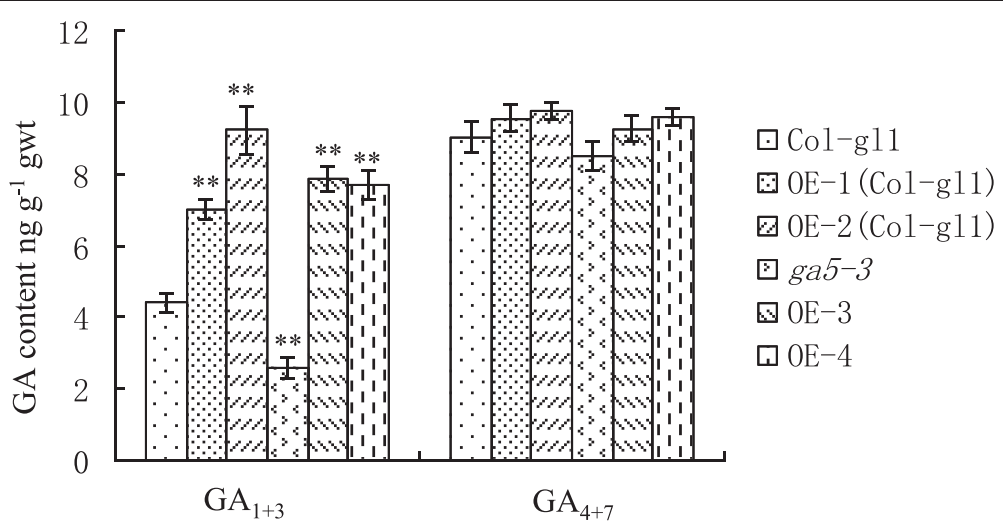

Fig. 5 The content of active gibberellin in the 7-d-old seedlings of transgenic and control plants. Each column represents the mean of three repeats with \pm SE bar. ${ }^{* *}$ means significant difference at 0.01 level

production phenotype was also found in $H v G A 20 o x_{2}$ transgenic Arabidopsis (Table 2, Figs. 3 and 4). The phenotype was characterized by elongated hypocotyl and stems, early flowering, and higher growth rate. In addition, barley GA20ox $x_{2}$ could recover $A t G A 20 o x_{1}$ loseof-function mutant. These results suggest that $H v G A 20 o x_{2}$ is the orthologous of $A t G A 20 o x_{1}$ and acts as the oxidase at carbon-20 of GA biosynthetic pathway.

The bioactive GAs in plants are $\mathrm{GA}_{1}, \mathrm{GA}_{3}, \mathrm{GA}_{4}$ and $\mathrm{GA}_{7}$, but $\mathrm{GA}_{4}$ is a major active GA in Arabidopsis [24]. Both endogenous $\mathrm{GA}_{1+3}$ and $\mathrm{GA}_{4+7}$ in 7-day-old seedlings of the control and transgenic plants were quantified in this study. The results showed that transgenic plants had higher GA contents, especially for $\mathrm{GA}_{1+3}$ compared with the control. The increased GAs is associated with the changed phenotype (Figs. 3 and 4). Similar results have been reported that $\mathrm{GA}_{1}$ content was increased and $\mathrm{GA}_{4}$ content had little change in the 7-day-

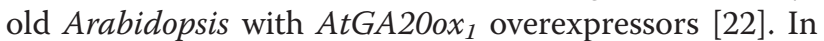
contrast, both $\mathrm{GA}_{1}$ and $\mathrm{GA}_{4}$ content had slight change in the shoot tip of Arabidopsis plant with overexpression AtGA20ox, while a 2- to 3-fold increase in $\mathrm{GA}_{4}$ content was observed in the rosette leaves of the transgenic lines [23]. Radi et al. [25] deduced that the dramatic difference in GAs content might be due to the variation in GA level among plant tissues and growth stage.

As indicated in Fig. 6, the transgenic lines overexpressing barley GA 20-oxidases showed a decrease in $A t G A 200 x_{1}, A t G A 200 x_{2}, A t G A 30 x_{1}$ and an increase in $A t G A 2 o x_{2}$ expression, while ga5-3 was just opposite in the expression of these genes, which could be attributed to feedback from an increased bioactive GAs in $H v G A 20 x_{2}$ over-expressing transgenic Arabidopsis or decreased bioactive GAs in ga5-3, respectively. A similar regulatory role has been proposed for AtGA3ox 1 , whose transcript level is also feedback-regulated in transgenic Arabidopsis of over-expressed GA20ox [23, 26]. No considerable change was detected in $A t G A 20 o x_{3}$ level of transgenic Arabidopsis and the control, suggesting that $A t G A 20 o x_{3}$ gene might be less sensitive than AtGA20ox 1 and $A t G A 2 O \mathrm{Ox}_{2}$ to the alteration of bioactive GAs.

However, not all over-expressing GA20ox plants displayed GA-overdose phenotype. In the case of CmGA20ox1, over-expression of Cucurbita maxima GA 20-oxidase in Arabidopsis resulted in accumulation of inactive $\mathrm{GA}_{25}$ and $\mathrm{GA}_{17}$, and reduction of $\mathrm{GA}_{4}$ content, which caused a slight decrease in stem elongation $[25,27]$. Accordingly, GAs accumulation and changed expression levels of GA-regulated transcripts confirmed that $H v G A 20 o x_{2}$ should be involved in regulation of GAs production. In barley semi-dwarf mutant Riso no. 9265, lose of HvGA20ox 2 caused its GA deficiency and reduced plant height.

It was reported that GA promoted stem elongation and was found in the young tissues [22, 23]. HvGA20ox 2 is highly expressed in stem and possiblely has effect on internode length [15]. Once jointing stage starts, the stems are young and internode region begins to elongate and grow rapidly. Therefore, the transcriptional levels of GA biosynthetic and catabolic genes were detected at the initial jointing stage. As a result, we found that GA biosynthesis genes (HvGA20ox 1 and $\left.H v G A 3 o x_{2}\right)$ were up-regulated and GA catabolic gene $\left(H v G A 2 o x_{3}\right)$ was down-regulated in Riso no. 9265 compared with Bomi, suggesting a type of feedback regulation from the low bioactive GAs because of the null mutation of $H v G A 2 O o x_{2}$. Feedback regulation of GA2Oox and GA3ox and feed-forward regulation of GA2ox genes expression have been also shown in GA-deficient Arabidopsis mutants [27-29]. In rice $s d 1$, the expression of OsGA20ox 1 was increased in stem to compensate the $s d 1$ effect $[5,30]$. As a result, the increased expression of $H \nu G A 20 o x_{1}$ could compensate the effect of $H \nu G A 20 o x_{2}$ in the stem at least partially, and the feedback or feed-forward regulation of the GA dioxygenase 

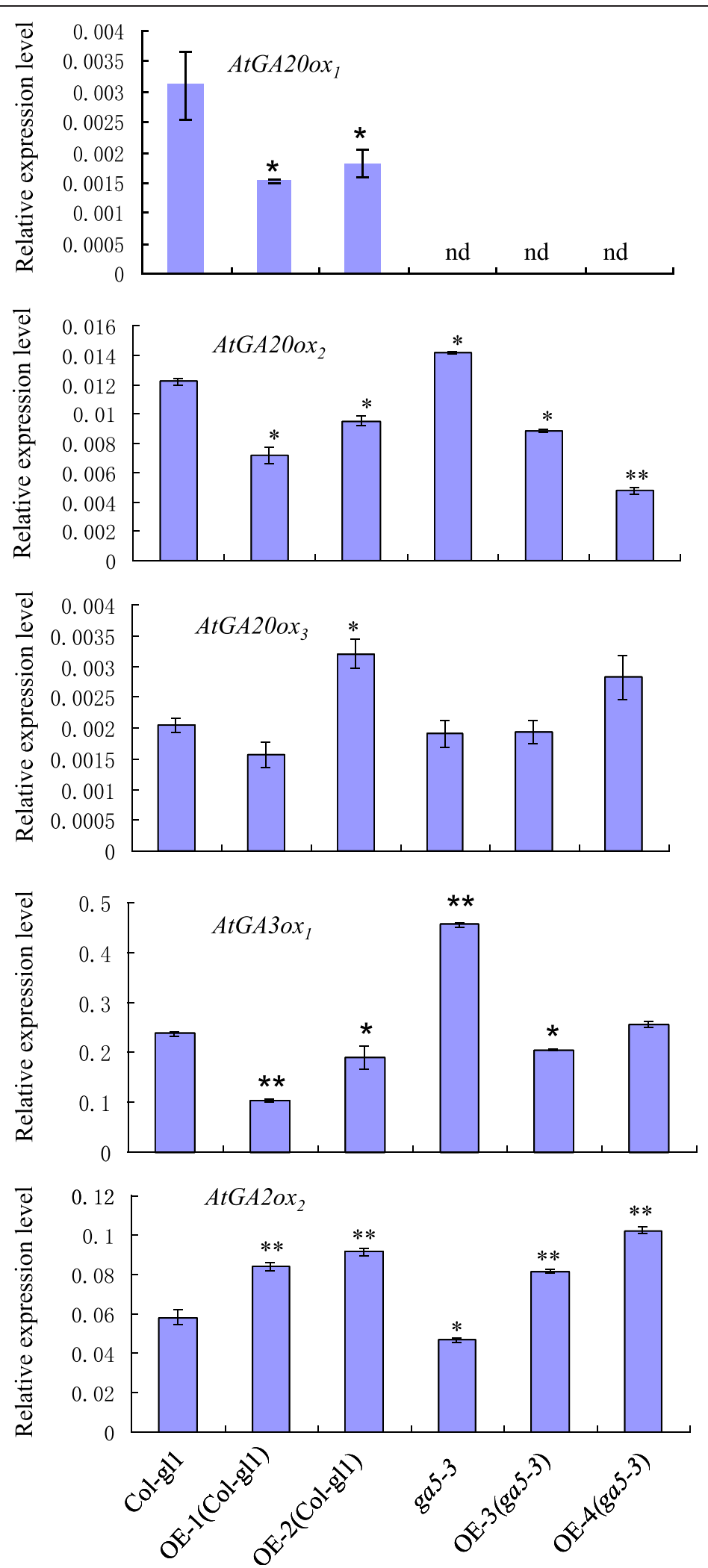

Fig. 6 The effect of over expression HvGA20ox on expression of gibberellin biosythetic and catabolic genes. *indicades significant difference at 0.05 level; **means significant difference at 0.01 level.; nd means not detected 


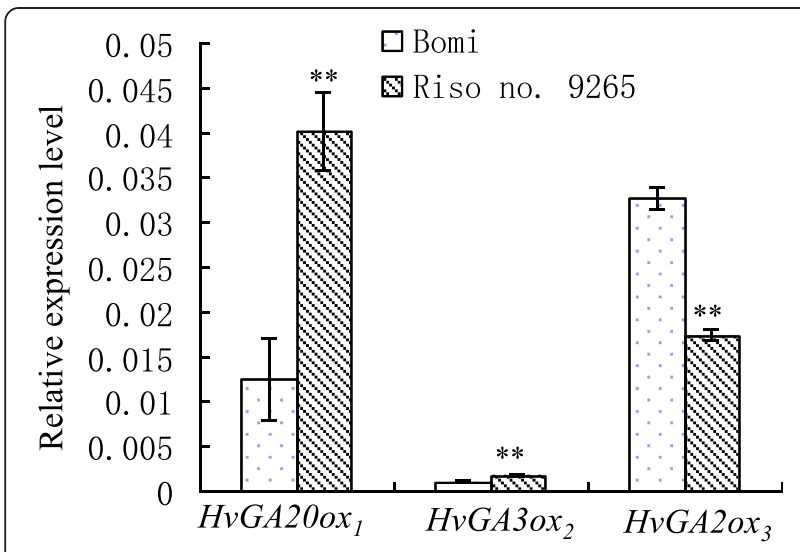

Fig. 7 Transcriptional changes of barley GA biosynthetic and catabolic genes in stem at the initial jointing stage. ${ }^{*}$ means significant difference at 0.01 level

helps maintaining a relative stable endogenous GA level. Consequently, Riso no. 9265 exhibited the observed plant height.

As mentioned above, we have characterized the barley semi-dwarf gene, $s d w 1 / d e n s o$, and conclude that it encodes a GA biosynthetic enzyme, GA20ox, on the basis of the following results. Firstly, sdw1/denso mutant responds to exogenous $\mathrm{GA}_{3}[15,18]$. Secondly, the fourth $s d w 1$ allele is a complete lose of HvGA20ox ${ }_{2}$ activity due to the deletion of $H v G A 20 o x_{2}$ gene in Riso no. 9265 (Fig. 1). In contrast, denso allele and $s d w 1$ of Jotun shows different expression of $H \nu G A 20 o x_{2}$ [15]. Thirdly, the transgenic wild type Arabidopsis and semi-dwarf mutant ga5-3 by HvGA20ox 2 gene showed GA over-dose phenotypes. Finally, GA-regulated transcripts were changed in Riso no. 9265 in the same way as those of some GA20oxs in rice and Arabidopsis [5, 23, 26, 30].

It was reported that $s d w 1 /$ denso was associated with later heading [11, 31]. Upon first mapped denso to the long arm of chromosome $3 \mathrm{H}$, Barua et al. [19] found a quantitative trait locus for heading date, which could not be genetically separated from the denso locus. Previously identified QTLs of heading date was also located on the region around denso in the Blenheim $\times \mathrm{E} 224 / 3 \mathrm{DH}$ population [14]. Furthermore, it was found that $s d w 1$ delayed maturity by around 3d based on eight populations [10]. We also found the QTL of development score was co-located with the HvGA20ox 2 eQTL on 3HL [15]. However, it is still difficult to distinguish if it is pleiotropy of $s d w 1 / d e n s o$ or a tight linkage between the gene and one controlling later heading. In the present study, over-expression of $H v G A 20 o x_{2}$ caused early flowering in Arabidopsis. The same is true for AtGA20oxs overexpressors [22, 23]. Both AtGA20ox $x_{1}$ and AtGA20ox 2 act redundantly and affect many developmental processes, with $A t G A 20 o x_{1}$ making the great contribution to internode and filament elongation, and $A t G A 20 o x_{2}$ making the great contribution to flowering time and silique length in Arabidopsis [29]. It was also revealed that either $A t G A 20 o x_{1}$ or $A t G A 20 o x_{2}$ mutation delayed flowering under short-day condition,while only $A t G A 20 o x_{2}$ delayed flowering slightly under long-day condition. Thus it can be assumed that the deficiency of $A t G A 20 o x_{1}$ or $A t G A 20 o x_{2}$ together with low GA affects flowering time, because GA acts a particularly important developmental switch between vegetative and reproductive development [32]. In the same way, it might be the deletion of $H \nu G A 20 o x_{2}$ that causes later heading due to low GA concentration in Riso no. 9265. The hypothesis could be proved by the following facts. Firstly, GAs is involved in many developmental processes, including germination, stem extension, flowering and fruit set $[22,23$, 29, 32]. Secondly, HvGA20ox 2 and Mloc_56463 are absent in Riso no. 9265, unlike its wild type, while it is $H \nu G A 20 o x_{2}$ that has gibberellin 20-oxidase activity and acts as a major determinant for GA production. Thirdly, GA deficiency was proved in $s d w 1 /$ denso using GA sensitive experiment [15, 18]. Lastly, Riso no. 9265 mutant exhibited a late heading date. Similar results were found that semi-dwarf progenies with $s d w 1 /$ denso matured generally later than their tall counterparts $[10,11,31]$. Considering all of these, it can be concluded that late heading in $s d w 1 / d e n s o$ could be the pleiotropy of $H v G A 20 o x_{2}$.

\section{Conclusions}

The current study showed a complete deletion of over $21 \mathrm{~kb}$ DNA fragment including both $\mathrm{HvGA2Oox}_{2}$ and Mloc_56463 genes in the mutant Riso no. 9265. $H v G A 20 o x_{2}$ acts as GA 20-oxidase and is involved in gibberellin biosynthesis. The expression of the genes encoding GA biosynthesis $\left(H \nu G A 20 o x_{1}\right.$ and $\left.H \nu G A 3 o x_{2}\right)$ are up-regulated and the expression of GA catabolic gene $H v G A 2 o x_{3}$ is down-regulated in Riso no. 9265 in comparison with those in wild type Bomi, respectively. We conclude that $s d w 1 /$ denso encodes one of the GA biosynthetic enzymes, GA 20-oxidase and the deletion of $H \nu G A 20 o x_{2}$ as well as the compensatory of $H v G A 20 o x_{1}$ and feedback regulation of gibberellin results in semidwarf phenotype of Riso no. 9265. We also deduced that $s d w 1 /$ denso allele itself evoked later heading due to its reduced endogenous GAs concentration.

\section{Methods}

\section{Plant materials and sampling}

Semi-dwarf mutant Riso no. 9265, kindly provided by Dr. Chengdao Li of Murdoch University, Australia and its wild parent Bomi, kindly provided by Dr. Jing Zhang of Chinese Academy of Agricultural Sciences, China were used in this study. Both genotypes were planted in $2 \times 6 \mathrm{~m}$ plots. The heading date was recorded as the 
number of days after sowed when $50 \%$ of the spike emerged from the sheath. At maturity, 20 plants of each genotype were harvested at random for measurement of plant height, spike and internode length. Meanwhile, main stems of each genotype were taken at the initial jointing stage for measuring the expression levels of gibberellin biosynthetic and catabolic genes.

\section{DNA isolation, PCR amplification and sequencing}

Seedlings at tillering stage were sampled and DNA was extracted according to Murray and Thompson [33] with small modification. DNA samples were quantified using a Thermo Unicam UV300 and adjusted to a final concentration of $50 \mathrm{ng} / \mu \mathrm{l}$ for PCR analysis. PCR was performed on a Veriti 96 well thermocycler (Applied Biosystems). Sequencing of $H v G A 200 x_{2}$ isolated from barley was conducted using two-pair primers: (1) forward primer (sdwF10) 5' CTAGCTCACACACCTCTCA TCTCAT 3', and reverse primer (sdwR10) 5' GTTCCCG ACAAAAATTCCGTGT 3'; (2) forward primer (sdwF9) 5' CTCTCCCGCACACTCACTCGCAAC 3', and reverse primer (sdwR13) 5' GCGGTGAGGGGGCATGCATAT 3'. PCR reaction was comprised of $50 \mathrm{ng}$ of template DNA, $0.3 \mu \mathrm{M}$ of each primer, $1 \times$ PCR buffer, $0.4 \mathrm{~mm}$ dNTP and 0.2 U PCR enzyme (KOD FX, TOYOBO) in a final volume of $10 \mu \mathrm{l}$. PCR cycling conditions consisted of an initial denaturation step of $94{ }^{\circ} \mathrm{C}$ for $3 \mathrm{~min}$, followed by $30-35$ cycles of $98{ }^{\circ} \mathrm{C}$ for $10 \mathrm{~s}, 60^{\circ} \mathrm{C}$ for $30 \mathrm{~s}$, and $68{ }^{\circ} \mathrm{C}$ for $3 \mathrm{~min}$.

Primers of Morex_contig_40861 and neighbor genes of $H v G A 20 o x_{2}$ were listed in Additional file 1: Table S1. PCR reaction was comprised of $1 \times$ Taq Mix (Bio life), $0.3 \mu \mathrm{M}$ of each primer and $50 \mathrm{ng}$ of template DNA. The following PCR amplification profile was used : denaturation at $94{ }^{\circ} \mathrm{C}$ for $3 \mathrm{~min}$, followed by 35 cycles of $94{ }^{\circ} \mathrm{C}$ for $30 \mathrm{~s}, 55{ }^{\circ} \mathrm{C}$ for $30 \mathrm{~s}, 72{ }^{\circ} \mathrm{C}$ for $0.5-2 \mathrm{~min}$ depending on the size of amplilcons, and a final extension at $72{ }^{\circ} \mathrm{C}$ for $5 \mathrm{~min}$. The amplification products were run in $1 \%$ agrose gels and sequenced by Biosune Biotechnology Co. Ltd.

\section{Plasmid construction and Arabidopsis transformation}

For plasmid construction, full-length CDS of $H v G A 20 o x_{2}$ was amplified from Bomi by PCR. The primers were designed as 5' AGTACTCGAGCTCACACACCTCTCAT CTCAT 3 ' and 5'CTATGGATCCGAATCAGCCCGTG GAT 3' with XhoI and BamH I sites, respectively. The amplified PCR product was confirmed by sequencing and cloned into T4 vector for further manipulation. Insert was confirmed by sequencing. The CDS paragraph of $H v G A 20 o x_{2}$ digested with $\mathrm{Xhol} / \mathrm{BamH} \mathrm{I}$ was cloned into the XhoI/ BamH Isite located between the 35S CaMV promoter and ocs terminator of the pFGC5941 binary vector, named as pFGC5941- HvGA20ox 2 . The binary vector was transformed into Agrobacterium tumefaciens by electroporation, and the floral dip method was used to transform Col-gl1 (Columbia ecotype, different from Colo only in its glabrous leaf. Hereafter refer to wild type) and a semi-dwarf mutant ga5-3 (a T-DNA inserted mutant of $A t G A 20 o x_{1}$ in Col-o, Salk016701) [34]. T1 transgenic plants were screened by spraying bialaphos solution twice at an interval of three days at cotyledon stage, and confirmed by PCR using following specific primers: Forward, 5' GGAGCATCGTGGAAAAAGAAGA 3' (from CaMV 35S promoter sequence) and Reverse, 5' GGAGTCG CAGGGCTGGTGTCC 3 '(from $H \nu G A 20 o x_{2}$ ). The constructs were also verified by sequencing. For analysis of $H \nu \mathrm{GA}_{20 \mathrm{O} x_{2}}$ gene expression level in transgenic plants, 7day-old Col-gl1, ga5-3 and T3 transgenic seedlings were harvested for RNA extraction.

\section{Growth conditions and phenotypic characterization of Arabidopsis}

The seeds of Arabidopsis were stratified at $4{ }^{\circ} \mathrm{C}$ for $1-2 \mathrm{~d}$ and sown on soil at $24{ }^{\circ} \mathrm{C}$ under LDs (16 h of light). Plants for hypocotyl and root length measurements were grown vertically on one half times Murashige and Skoog media (Sigma, M6899) containing 1 \% MES, 3 \% sucrose and $0.46 \%$ Gelrite with $\mathrm{pH} 5.8$ under LDs, being measured after $7 \mathrm{~d}$. Flowering time was scored as the days when buds could be detected with naked eyes. Other measurements were performed on the plants that had stopped flowering.

\section{Real-time quantitative RT-PCR}

RNA was extracted from the stems of Bomi and Riso no. 9265, as well as 7-day-old seedlings of T3 transgenic lines, Col-gl1 and ga5-3 using Spin Column Plant total RNA Purification Kit(Sanggon Biotech (Shanghai) Co., Ltd). cDNA was prepared from $1 \mu \mathrm{g}$ RNA using AMV First Strand cDNA Synthesis Kit(Sanggon Biotech (Shanghai) Co., Ltd). qPCR reactions were performed using SYBR Green (SG Fast qPCR Master Mix(High Rox), BBI ) and the Applied Biosystems Stepone plus Real-time PCR System. The Real-time PCR assays were performed in triplicate for each cDNA sample. For determining transcription levels of barley GA20ox 2 and genes encoding final biosynthesis of GA, HVACTIN and HvGAPDH for barley, and At1g13320 and At4g26410 for Arabidopsis were employed as reference genes $[5,35,36]$. Additional file 2: Table S2 listed the oligonuleotide sequences used for quantitative RT-PCR.

\section{ELISA assay of gibberellin $\left(\mathrm{GA}_{1+3}\right.$ and $\left.\mathrm{GA}_{4+7}\right)$}

Approximately $0.5 \mathrm{~g}$ fresh Arabidopsis tissue (7-day-old seedling) was homogenized in liquid nitrogen and extracted at $4{ }^{\circ} \mathrm{C}$ in cold $80 \%(\mathrm{v} / \mathrm{v})$ methanol containing $1 \mathrm{mM}$ butylated hydroxytoluene for $4 \mathrm{~h}$. The extracts 
were collected after centrifugation at $8000 \mathrm{~g}$ at $4{ }^{\circ} \mathrm{C}$ for $20 \mathrm{~min}$. The residues were suspended in the same extraction solution and stored at $4{ }^{\circ} \mathrm{C}$ for $1 \mathrm{~h}$, and then centrifuged again at $8000 \mathrm{~g}$ at $4{ }^{\circ} \mathrm{C}$ for $20 \mathrm{~min}$. The two resulting supernatants were combined and passed through a $\mathrm{C}_{18}$ Sep-Pak cartridge (Waters, Milford, MA, USA). The efflux was collected and dried in $\mathrm{N}_{2}$. The residues was then dissolved in $0.01 \mathrm{M}$ phosphate buffer solution ( $\mathrm{pH}$ 7.5) and concentrations of $\mathrm{GA}_{1+3}$ and $\mathrm{GA}_{4+7}$ were determined in an indrect enzyme-linked immuno sorbent assay (ELISA) using $\mathrm{GA}_{3}$ and $\mathrm{GA}_{4}$ antibody, respectively. The ELISA KIT was obtained from Professor Baomin Wang (Chinese Agricultural University) and the methods were described in previous publications $[37,38]$.

\section{Statistical analyses}

Phenotypic differences of Bomi and Riso no. 9265 were tested by Student's $t$ test. In order to avoid heterogeneity of variance, a natural log transformation was applied to hypocotyl length,and a square transformation was applied to flowering time (days), plant height, root and internode length. Least significant difference (LSD) at $5 \%$ probability was used to assess the difference between genotypes. For statistical analysis of qPCR data, cycle threshold $\left(C_{T}\right)$ values were used to determine $\Delta$ $C_{T}$ values $\left(\Delta C_{T}=C_{T}\right.$ target $-C_{T}$ reference $)$, and expression levels of target genes relative to reference gene were determined as $2^{-\Delta} \mathrm{CT}$. For comparison of GA concentration and the expression levels of GA-regulated transcripts between transgenic and wild type plants, ANOVA was used. Analysis of qRT-PCR efficiency showed that all amplicons of the genes used in this study were within the optimal range of 98.7-102.4\%.

\section{Ethics Statement}

In this study we only used plant material including barley and Arabidopsis. Based on the rule of BMC Genomics, no ethics statement was required for the collection of genetic material.

\section{Additional files}

Additional file 1: Table S1. Primers used to detect flanking genes or sequences of HVGA2OOX. (XLS $18 \mathrm{~kb}$ )

Additional file 2: Table S2. Oligonucleotide sequences used in qRT-PCR assays. (XLS $17 \mathrm{~kb}$ )

\section{Competing interests}

The authors declare that they have no competing interests.

\section{Authors' contributions}

QJ, CL, JY and GZ made the experimental design. QJ and YS performed to identify the deleted sequences and GRT-PCR GA related genes. QJ and JZ were involved in phenotyping Bomi and Riso no. 9265. QJ and WH developed the transgenic Arabidopsis and characterized phenotype of Arabidopsis. QJ, JZ and JW conducted statistical analysis of the expression data and phenotypic data. QJ, CL and GZ wrote the manuscript, and all authors read and approved the final version.

\section{Acknowledgements}

We thank Dr Xuelong Wu (Institute of Virology and Biotechnology, Zhejiang Academy of Agricultural Sciences, China) for technical assistance in plasmid constructs and Arabidopsis transformation. This work was supported by National Natural Science Foundation of China (Grant No. 31471495), Science and Technology Innovation Team Project of Zhejiang Province (2011R50026-9), China Agriculture Research System (CARS-05) and Young talent project of Zhejiang Academy of Agricultural Sciences.

\section{Author details}

'Institute of Crop Science, Zhejiang University, Hangzhou 310058, China. ${ }^{2}$ Institute of Crop and Nuclear Technology Utilization, Zhejiang Academy of Agricultural Sciences, Hangzhou 310021, China. ${ }^{3}$ Western Barley Genetics Alliance, Murdoch University, Murdoch WA6150, Australia.

Received: 22 July 2015 Accepted: 20 October 2015

Published online: 14 November 2015

\section{References}

1. Zapata TC, Silva CP, Acevedo HE. Grain yield and assimilate partitioning in wheat isogenic plant height lines. Agric Tec. 2004;64(2):139-55.

2. Khush GS. Green revolution: preparing for the 21st century. Genome. 1999:42(4):646-55.

3. Ashikari M, Sasaki A, Ueguchi-Tanaka M, Itoh H, Nishimura A, Datta S, et al. Loss-of-function of a rice gibberellin biosynthetic gene, GA20 oxidase (GA200x-2), led to the rice 'green revolution'. Breed Sci. 2002;52:143-50.

4. Monna L, Kitazawa N, Yoshino R, Suzuki J, Masuda H, Maehara Y, et al. Positional cloning of rice semidwarfing gene, $s d-1$ : rice "green revolution gene" encodes a mutant enzyme involved in gibberellin synthesis. DNA Res. 2002;9:11-7.

5. Sasaki A, Ashikari M, Ueguchi-Tanaka M, Itoh H, Nishimura A, Swapan D, et al. Green revolution: a mutant gibberellin-synthesis gene in rice. Nature. 2002:416:701-2.

6. Spielmeyer W, Ellis MH, Chandler PM. Semidwarf (sd-1), green revolution rice, contains a defective gibberellin 20-oxidase gene. Proc Natl Acad Sci U S A. 2002;99:9043-8.

7. Kuczyńska A, Surma M, Adamski T, Mikołajczak K, Krystkowiak K, Ogrodowicz P. Effects of the semi-dwarfing sdw1/denso gene in barley. J Appl Genetics. 2013;54:381-90.

8. Jia Q, ZHang J, Westcott S, Zhang X, Bellgard M, Lance R, et al. GA-20 oxidase as a candidate for the semidwarf gene sdw1/denso in barley. Funct Integr Genomics. 2009;9:255-62.

9. Barboza L, Effgen S, Alonso-Blanco C, Kooke R, Keurentjes JJ, Koornneef M, et al. Arabidopsis semidwarfs evolved from independent mutations in GA200x1, ortholog to green revolution dwarf alleles in rice and barley. Proc Natl Acad Sci U S A. 2013;110(39):15818-23.

10. Hellewell KB, Rasmusson DC, Gallo-Meagher M. Enhancing yield of semidwarf barley. Crop Sci. 2000;40:352-8.

11. Thomas WTB, Powell W, Swanston JS. The effects of major genes on quantitatively varying characters in barley. 4. The GPert and denso loci and quality characters. Heredity. 1991;66:381-9.

12. Mickelson HR, Rasmusson DC. Genes for short stature in barley. Crop Sci. 1994;34:1180-3.

13. Thomas WTB, Powell W, Waugh R, Chalmers K, Barua UM, Jack $P$, et al. Detection of quantitative trait loci for agronomic, yield, grain and disease characters in spring barley (Hordeum vulgare L.). Theor Appl Genet. 1995;91:1037-47

14. Powell W, Thomas WTB, Baird E, Lawrence P, Booth A, Harrower B, et al. Analysis of quantitative traits in barley by the use of amplified fragment length polymorphisms. Heredity. 1997;79:48-59.

15. Jia Q, Zhang XQ, Westcott S, Broughton S. CakirM, Yang J, et al: Expression level of a gibberellin 20-oxidase gene is associated with multiple agronomic and quality traits in barley. Theor Appl Genet. 2011;122:1451-60

16. Grausgruber H, Bointner H, Tumpold R, Ruckenbauer P. Genetic improvement of agronomic and qualitative traits of spring barley. Plant Breed. 2002;121:411-6.

17. Haahr V, von Wettstein D. Studies of induced, high yielding dwarf-mutant of spring barley. In: Gaul H, editor. Barley Genetics III.: Proceedings of the 
3rd International Barley Genetics Symposium. München: Verlag Karl Thiemig; 1976. p. 215-8.

18. Franckowiak JD, Pecio A. Coordinator's report: a listing of genetic stocks Barley Genet Newsl. 1992;21:116-26.

19. Barua UM, Chalmers KJ, Thomas WTB, Hackett CA, Lea V, Jack $P$, et al. Molecular mapping of genes determining height, time to heading, and growth habit in barley (Hordeum vulgare). Genome. 1993;36:1080-7.

20. Mayer K, Waugh R, Brown J, Schulman A, Langridge P, Platzer M, et al. A physical, genetic and functional sequence assembly of the barley genome. Nature. 2012;491:711-6.

21. Hedden P, Phillips AL. Gibberellin metabolism: new insights revealed by the genes. Trends Plant Sci. 2000;5:523-30.

22. Huang S, Raman AS, Ream JE, Fujiwara H, Cerny RE, Brown SM. Overexpression of 20-oxidase confers a gibberellin-overproduction phenotype in Arabidopsis. Plant Physiol. 1998;118(3):773-81.

23. Coles JP, Phillips AL, Croker SJ, García-Lepe R, Lewis MJ, Hedden P. Modification of gibberellin production and plant development in Arabidopsis by sense and antisense expression of gibberellin 20-oxidase genes. Plant J. 1999;17(5):547-56.

24. Talon M, Koornneef M, Zeevaart JAD. Endogenous gibberellins in Arabidopsis thaliana and possible steps blocked in the biosynthetic pathways of the semidwarf ga4 and ga5 mutants. Proc Natl Acad Sci U S A. 1990;87:7983-7.

25. Radi A, Lamge T, Niki T, Koshioka M, Lange MJP. Ectopic expression of pumpkin gibberellin oxidases alters gibberellin biosynthesis and development of transgenic Arabidopsis plants. Plant Physiol. 2006;140(2):528-36.

26. Carrera E, Bou J, García-Martínez JL, Prat S. Changes in GA 20-oxidase gene expression strongly affect stem length, tuber induction and tuber yield of potato plants. Plant J. 2000;22(3):247-56.

27. $\mathrm{Xu} Y \mathrm{Y}, \mathrm{Li}$ L, Gage DA, Zeevaart JA. Feedback regulation of GA5 expression and metabolic engineering of gibberellin levels in Arabidopsis. Plant Cell. 1999;11(5):927-36.

28. Phillips AL, Ward DA, Uknes S, Appleford NE, Lange T, Huttly AK, et al. Isolation and expression of three gibberellin 20-Oxidase cDNA clones from Arabidopsis. Plant Physiol. 1995;108:1049-57.

29. Rieu I, Ruiz-Rivero O, Fernandez-Garcia N, Griffiths J, Powers SJ, Gong F, et al. The gibberellin biosynthetic genes AtGA200x1 and AtGA20ox2 act, partially redundantly, to promote growth and development throughout the Arabidopsis life cycle. Plant J. 2008;53:488-504.

30. Asano K, Takashi T, Miura K, Qian Q, Kitano H, Matsuoka M, et al. Genetic and molecular analysis of utility of sd1 alleles in rice breeding. Breed Sci. 2007:57:53-8.

31. Powell W, Caligari PDS, Thomas WTB, Jinks JL. The effects of major genes on quantitatively varying characters in barley. 2 . The denso and daylength response loci. Heredity. 1985;54:349-52.

32. Eriksson S, Bohlenius H, Moritz T, Nilsson O. GA4 is the active gibberellin in the regulation of LEAFY transcription and Arabidopsis floral initiation. Plant Cell. 2006;18:2172-81.

33. Murray MG, Thompson WF. Rapid isolation of high molecular weight plant DNA. Nucl Acids Res. 1980;8(19):4321-5.

34. Clough JP, Bent AF. Floral dip: a simplified method for Agrobacteriummediated transformation of Arabidopsis thaliana. Plant J. 1998;16(6):735-43.

35. Boden SA, Weiss D, Ross JJ, Davies NW, Trevaskis B, Chandler PM, et al. EARLY FLOWERING3 regulates flowering in spring barley by mediating gibberellin production and FLOWERING LOCUS T expression. Plant Cell. 2014;26:1557-69.

36. Czechowski T, Stitt M, Altmann T, Udvardi MK, Scheible WR. Genome-wide identification and testing of superior reference genes for transcript normalization in Arabidopsis. Plant Physiol. 2005;139:5-17.

37. Yuan Y, Liu Y, Wu C, Chen S, Wang Z, Yang Z, et al. Water deficit affected flavonoid accumulation by regulating hormone metabolism in scutellaria baicalensis georgi roots. Plos One. 2012;7(10):e42946. doi:10.1371/journal.pone.0042946.

38. Jia YX, Tao FQ, Li WQ. Analysis of endogenous hormone levels to reveal the retardation by suppression of phospholipase Da1 in Arabidopsis leaves during hormone-promoted senescence. Plant Diversity Res. 2014;36(4):485-96

\section{Submit your next manuscript to BioMed Central and take full advantage of:}

- Convenient online submission

- Thorough peer review

- No space constraints or color figure charges

- Immediate publication on acceptance

- Inclusion in PubMed, CAS, Scopus and Google Scholar

- Research which is freely available for redistribution

Submit your manuscript at www.biomedcentral.com/submit 